\title{
Chance für Kooperation
}

\section{Mit Hilfe von Ökobilanzen soll Orientierungswissen für politische und wirt- schaftliche Akteure gewonnen werden. Fraglich ist allerdings, wieweit sie direkte politische Eingriffe in die Wirtschaft legitimieren können.}

\begin{abstract}
I hre zentrale Rolle spielen Ökobilanzen bei der Gestaltung von Produkten und Verfahren durch die Unternehmen: Sie identifizieren ökologische Schwachstellen gegenwärtiger Produkte, sie erschließen Ansatzpunkte für ökologische Verbesserungen und machen die Gestaltungsparameter für deren Verwirklichung sichtbar. Ökobilanzen fördern damit dezentrale Innovationen in Sachen Umweltschutz.
\end{abstract}

\section{Ökobilanzen zur Orientierung}

Ökobilanzen können zugleich Teilinformationen zur umweltpolitischen „Landkarte“ beitragen, also „Orientierungswissen“ für politische und wirtschaftliche Akteure liefern. Fraglich ist aber, inwieweit Ökobilanzen hierüber hinaus direkte politische Eingriffe legitimieren können, die nicht nur - wie das etablierte Umweltrecht - auf bestimmte Formen der Inanspruchnahme der Umwelt, Ressourcen, Umweltmedien usw. gerichtet sind, sondern direkt auf einzelne Produkte bzw. auf die $\mathrm{zu}$ ihrer Herstellung genutzten Prozesse. Ein Beispiel ist die Verpackungspolitik: Mit diesem kategorial neuen Ansatz würden vergleichende Ökobilanzen die Legitimationsgrundlage für politische Maßnahmen zugunsten der „Gewinner“" und zu Lasten der „Verlierer“. Die Rolle, die Ökobilanzen in der Diskussion um die Verpackungspolitik, namentlich bei der Begründung der Mehrwegquote, spielen (Stichwort „Dosen gegen Mehrwegflaschen") wird von vielen in der Industrie als erstes Beispiel für eine solche Politik gesehen. Dies birgt erhebliche Risiken - ökonomische, ökologische und politische: Anstatt technologische Optionen $\mathrm{zu}$ identifizieren, bei denen die Unternehmen nach Maßgabe ihrer spezifischen Bedingungen den höchsten ,ökologischen Grenznutzen" bei ihren Investitionen erreichen, würden bestehende Produktionskapazitäten und Marktanteile in Frage gestellt. Anstatt zu einer höheren ökologischen Treffsicherheit von Investitionen beizutragen, würden Ökobilanzen somit zum ökonomischen Risikofaktor. Die Entscheidung für eine Technologie müßte sich neben dem Risiko des Marktes auch noch demjenigen der staatlichen „Produktbewertung“ aussetzen - mit einander möglicherweise direkt widersprechenden Ergebnissen. Umgekehrt würden derartige Ökobilanzen um so mehr zum Austragungsort konkurrierender wirtschaftlicher Interessen, wie sie - je nach Interessenlage - als potentielles „Korrektiv“ des Marktes zu wirken versprechen bzw. drohen.

Die wesentliche Leistung der Marktwirtschaft, nämlich Suchprozesse nach besseren Problemlösungen $z u$ initiieren, würde ebenso konterkariert wie der Hauptzweck von Ökobilanzen, ökologische Verbesserungspotentiale im Lebensweg aufzudecken. Einerseits würde die Chance, daß die „Verlierer" ihre Technologien ökologisch optimieren, nicht genutzt. Andererseits sänke für die „Gewinner" der Anreiz weiterer Innovationen in dem Maße, wie die Politik den Marktanteil der „Verlierer" durch Quoten begrenzt, ihre Preise durch Steuern erhöht, oder den Markt für ausländische Importe abschottet. Zum Beispiel hätte der ökologisch hochgradig positive Wettbewerb zwischen Stahl, Aluminium und Kunststoff im Automobilbau kaum stattgefunden, wenn die Politik schon vor zehn Jahren die jeweiligen Anteile quotiert hätte.

Eine solche Nutzung von Ökobilanzen wäre tendenziell auch für die Politik um so problematischer, je stärker einmalige Bewertungen gegen die Veränderung (relativer) ökologischer Leistungen von Technologien ,immunisiert" würden. Mit jedem Gesetz und jeder Verordnung, die mit einer Ökobilanz legitimiert würde, stiege die politische Schwelle, einmal identifizierte ,Gesetzmäßigkeiten" in Frage zu stellen. Je mehr umgekehrt ökologischer Wettbewerb wirksam wird und ggf. die jeweiligen Vor- und Nachteile verändert, desto fragwürdiger muß eine derartige Politik werden. Auch ist fraglich, in welchem Umfang sich die „relative ökologische Vorteilhaftigkeit“ verschieben muß, bis einmal geschaffene Förderund Diskriminierungsinstrumente in Frage gestellt würden. Die Politik begibt sich letztlich in ein Dilemma: Zur „Geisel“ einer ihrer Natur nach hochgradig brïchigen Legitimation $\mathrm{zu}$ werden oder aber heute ,zwingende“ Benachteiligungen und Vergünstigungen gegebenenfalls morgen zur Disposition zu stellen.

\section{Überfordertes Instrument}

Die Abstïtzung von relativ starrem Umweltrecht in Ökobilanzen und die damit verbundene Gefahr politischer, ökonomischer und ökologischer „Einbahnstraßen“ steht also in einem prinzipiellen Konflikt zu dem Grundanliegen, diskussionsund kritikfähige, veränderbare und Veränderung bewirkende Ergebnisse zu produzieren. Mit dem Anspruch, in der Zukunft wirksame Interventionen zu rechtfertigen, würde die Politik ein Instrument überfordern, das nicht mehr liefern kann und soll als Momentaufnahmen der Vergangenheit und Anstöße für künftige Innovationen.

Um Ökobilanzen für eine innovations- und nicht interventionsorientierte Politik zu nutzen, sind demgegenüber drei Voraussetzungen wichtig:

- Sie sollten darauf zielen, die wesentlichen technischen Einflußgrößen von Umweltbelastungen und technologische Verbesserungspotentiale zu identifizieren.

- Die wichtigen umweltpolitischen Ziele sollten die berechenbare Basis sein, an der sich Unternehmen bei der produkt- und prozeßspezifischen Nutzung von Ökobilanzen orientieren können.

- Ökobilanzen sollten als transparenter kooperativer Lernprozeß verstanden und organisiert werden - nicht als politisch-ökologisches „Gerichtsverfahren“.

Die Normenreihe ISO $14040 \mathrm{ff}$. bietet die beste Gewähr für eine kooperative Durchführung von Ökobilanzen, die zu transparenten, nachvollziehbaren Ergebnissen führt. Auf Basis dieser Norm sollten klare „Rechte und Pflichten“ für solche Studien erarbeitet werden, die für die Politik erstellt werden. Namentlich Einvernehmen über die Ziele einer Ökobilanz für die Politik herzustellen und mit Hilfe eines „Critical Review“ eine laufende „Qualitätskontrolle“ sicherzustellen, sind die Hauptvoraussetzungen dafür, durch von der Politik initiierte Ökobilanzen Innovationsprozesse in Gang zu setzen.

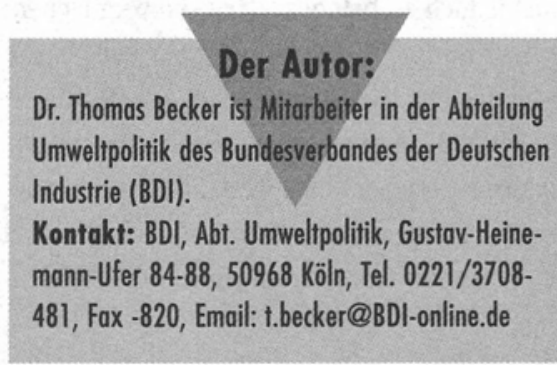


(c) 20I0 Authors; licensee IÖW and oekom verlag. This is an article distributed under the terms of the Creative Commons Attribution Non-Commercial No Derivates License (http://creativecommons.org/licenses/by-nc-nd/3.o/), which permits unrestricted use, distribution, and reproduction in any medium, provided the original work is properly cited. 